\title{
OPTIMAL CONSUMPTION AND SLUTSKY EQUATION WITH EPSTEIN-ZIN TYPE PREFERENCE
}

\author{
SE RYOONG AHN ${ }^{1 \dagger}$ AND HYENG KEUN KOO ${ }^{2}$ \\ ${ }^{1}$ Department of Business Administration, Ajou University, South Korea \\ E-mail address: srahn@ajou.ac.kr \\ ${ }^{2}$ Department of Financial Engineering, Ajou University, South Korea \\ E-mail address: hkoo@a jou.ac.kr
}

\begin{abstract}
In this paper we conduct comparative statics for optimal consumption and portfolio selection of an agent who has a utility function of Epstein and Zin type. We derive the Slutsky equations and decompose the total effects of changes into the substitution effects and the income effects. We identify the role of the elasticity of intertemporal substitution and the coefficient of relative risk aversion.
\end{abstract}

\section{INTRODUCTION}

It has been long noted that portfolio selection is closely related to consumer choice. An economic agent finances future consumption by investing in assets. Two different allocations of investments will result in different consumption profiles for the agent. Thus, one can apply consumer choice theory to the study of portfolio selection. Pye [1] noted the importance of this relationship and hinted at the applicability of the Slutsky equation of consumer choice theory to comparative statics analysis for portfolio selection problems. Bierwag and Grove [2], Sandmo [3, 4], Fisher [5], Dalal [6], and Eichner [7, 8] have derived Slutsky equations for asset demands.

Despite the extensive literature on the subject, most research has been concerned with a static portfolio selection problem, ignoring intertemporal allocation of consumption. Fisher [5] was an exception and considered the intertemporal contingent consumption choice problem. In this paper we extend his analysis to the case where the economic agent has a recursive utility function of the Epstein-Zin type [9] and derive Slutsky equations for consumption and asset demand. This utility function allows separation of the elasticity of intertemporal substitution and the coefficient of relative risk aversion. This feature allows us to identify the roles of

\footnotetext{
Received by the editors March 19 2012; Accepted March 282012.

2000 Mathematics Subject Classification. 93B05.

Key words and phrases. Optimal consumption, Slutsky equation, Epstein-Zin utility.

${ }^{\dagger}$ Corresponding author.

The work is supported by the WCU (World Class University) Program through the Korea Science and Engineering Foundation funded by the Ministry of Education, Science and Technology (R31-20007). We thank Byung Lim Koo and Jung Lim Koo for helpful assistance.
} 
elasticity of intertemporal substitution and the coefficient of relative risk aversion on the overall effect of the income and substitution effects.

This paper is organized as follows. In section 2 we describe our model and solve our model in section 3. Section 4 contains analysis of the substitution and income effects and section 5 concludes.

\section{THE MODEL}

We consider a consumption and portfolio selection problem of an economic agent in a 2period discrete-time model. There exist two possible states of the world, $u$ and $d$, at time 1 .

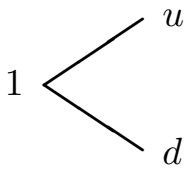

Stock

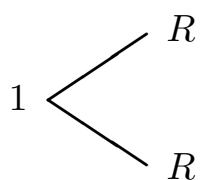

Bond $\pi_{u}$

$\pi d$

Probabilities

Figure 1. Asset Dynamics

The agent has the following utility function of the Epstein-Zin type:

$$
U\left(c_{0}, c_{1}\right)=\left[u\left(c_{0}\right)+\beta u\left(\mathbb{E}\left[\mu\left(\tilde{c}_{1}\right)\right]^{\frac{1}{\alpha}}\right)\right]^{\frac{1}{\rho}}
$$

where

$$
\begin{aligned}
& u(c)=c^{\rho}, \\
& \mu(c)=c^{\alpha} .
\end{aligned}
$$

$\rho<1, \alpha<1, \rho \neq 0, \alpha \neq 0, c_{0}$ is the agent's consumption at time $0, c_{u}$ and $c_{d}$ are her consumptions at state $u$ and $d$ at time 1 , respectively, and $\mathbb{E}$ is her expectation at time $0 .{ }^{1}$ It is well-known that the Epstein-Zin utility function has a constant elasticity of intertemporal substitution (EIS) equal to $\sigma \triangleq \frac{1}{1-\rho}$ and a coefficient of relative risk aversion (CRRA) equal to $\gamma \triangleq 1-\alpha$.

$$
\begin{aligned}
& { }^{1} \text { If } \rho=0 \text {, the utility function takes the form: } \\
& \qquad U\left(c_{0}, c_{1}\right)=\log c_{0}+\beta \log \left(\mathbb{E}\left[\mu\left(\tilde{c}_{1}\right)\right]^{\frac{1}{\alpha}}\right) .
\end{aligned}
$$

If $\alpha=0$, the utility function takes the form:

$$
U\left(c_{0}, c_{1}\right)=\left[u\left(c_{0}\right)+\beta u\left(\exp \left(\mathbb{E} \log \left(\tilde{c}_{1}\right)\right)\right)\right]^{\frac{1}{\rho}},
$$

where $u(c)=c^{\rho}$. The case $\rho=0$ or $\alpha=0$ can be regarded as the limiting case of the utility function in equation (2.1). We will derive our results based on this utility function. All our results, however, can be extended to the case $\rho=0$ or $\alpha=0$. 
The investment opportunity consists of a stock and a bond. The stock price at time 0 is normalized to be 1 and becomes $u$ in state $u$ and $d$ in state $d$ with $u>d .^{2}$ The bond is risk-free with an interest rate equal to $r$. Thus, the risk-free return, $R$, is equal to $1+r$. We assume a frictionless market. That is, there are no trading costs, taxes, and short-selling restrictions.

The agent wishes to maximize her utility given in (2.1) with her initial wealth $W$, choosing her consumption, $c_{0}, c_{u}, c_{d}$, and her investments, $\theta_{s}$ in the stock and $\theta_{b}$ in the bond. Her value function (indirect utility function) is defined as follows:

$$
V(W) \triangleq \max _{\{c\}, \theta_{s}, \theta_{b}}\left[\left(c_{0}\right)^{\rho}+\beta\left\{\pi_{u}\left(c_{u}\right)^{\alpha}+\pi_{d}\left(c_{d}\right)^{\alpha}\right\}^{\frac{\rho}{\alpha}}\right]^{\frac{1}{\rho}}
$$

subject to the budget constraints

$$
\begin{aligned}
& c_{0} \leq W-\theta_{s}-\theta_{b}, \\
& c_{u} \leq \theta_{s} u+\theta_{b} R, \\
& c_{d} \leq \theta_{s} d+\theta_{b} R .
\end{aligned}
$$

The financial market is complete, since there exist two possible future states and two assets with linearly independent payoffs. Thus, we can rewrite (2.2) - (2.4) as the following one constraint,

$$
c_{0}+p_{u} c_{u}+p_{d} c_{d} \leq W
$$

where

$$
\begin{aligned}
& p_{u}=\frac{R-d}{(u-d) R}, \\
& p_{d}=\frac{u-R}{(u-d) R} .
\end{aligned}
$$

Here $p_{u}$ and $p_{d}$ are equal to the prices of the Arrow-Debreu securities for states $u$ and $d$, respectively. Notice that because of the market completeness the problem is essentially that of consumption choices and portfolio selection is a consequence of the consumption choices.

\section{INDIVIDUAL CHOICE}

The agent's problem can be stated as follows:

Problem: The Agent's Consumption and Portfolio Choice Problem

The agent chooses her consumption bundle, $\left\{c_{0}^{1}, c_{u}^{1}, c_{d}^{1}\right\}$ and portfolio holdings, $\left\{\theta_{s}, \theta_{b}\right\}$, to maximize her utility given by (2.1) subject to budget constraint in (2.5).

The Lagrangian of the problem is

$$
\mathcal{L}=\left[\left(c_{0}\right)^{\rho}+\beta\left\{\pi_{u}\left(c_{u}\right)^{\alpha}+\pi_{d}\left(c_{d}\right)^{\alpha}\right\}^{\frac{\rho}{\alpha}}\right]^{\frac{1}{\rho}}+\lambda\left\{W-c_{0}-p_{u} c_{u}-p_{d} c_{d}\right\}
$$

\footnotetext{
${ }^{2}$ Here we use the same notation for the stock return and the state with a slight abuse of notation. The same notation for two different objects is for convenience and will not generate confusion.
} 
The first-order conditions are given as follows

$$
\begin{aligned}
& \frac{\partial \mathcal{L}}{\partial c_{0}}=\frac{\partial U}{\partial c_{0}}-\lambda=0, \\
& \frac{\partial \mathcal{L}}{\partial c_{i}}=\frac{\partial U}{\partial c_{i}}-p_{i} \lambda=0, i=u, d, \\
& \frac{\partial \mathcal{L}}{\partial \lambda}=W-c_{0}-p_{u} c_{u}-p_{d} c_{d}=0 .
\end{aligned}
$$

From (3.2) we get

and thus

$$
\frac{\frac{\partial U}{\partial c_{u}}}{\frac{\partial U}{\partial c_{d}}}=\frac{\pi_{u}}{\pi_{d}}\left(\frac{c_{u}}{c_{d}}\right)^{\alpha-1}=\frac{p_{u}}{p_{d}}
$$

$$
\frac{c_{u}}{c_{d}}=\left(\frac{\pi_{d}}{\pi_{u}} \frac{p_{u}}{p_{d}}\right)^{\frac{1}{\alpha-1}} \triangleq\left(\frac{\zeta_{u}}{\zeta_{d}}\right)^{\frac{1}{\alpha-1}}
$$

where $\zeta_{i}$ is the state price density of state $i$,

$$
\zeta_{i}=\frac{p_{i}}{\pi_{i}}
$$

And from (3.1) and (3.2),

$$
\begin{aligned}
p_{d} & =\frac{1}{\left(c_{0}\right)^{\rho-1}} \cdot \beta \pi_{d}\left(c_{d}\right)^{\alpha-1}\left\{\pi_{u}\left(\frac{\zeta_{u}}{\zeta_{d}}\right)^{\frac{\alpha}{\alpha-1}}\left(c_{d}\right)^{\alpha}+\pi_{d}\left(c_{d}\right)^{\alpha}\right\}^{\frac{\rho}{\alpha}-1} \\
\Rightarrow \frac{c_{d}}{c_{0}} & =\beta^{\frac{1}{1-\rho}} \zeta_{d}^{\frac{1}{\alpha-1}}\left(\pi_{u} \zeta_{u}^{\frac{\alpha}{\alpha-1}}+\pi_{d} \zeta_{d}^{\frac{\alpha}{\alpha-1}}\right)^{\frac{\rho-\alpha}{\alpha(1-\rho)}} .
\end{aligned}
$$

Symmetrically,

$$
\frac{c_{u}}{c_{0}}=\beta^{\frac{1}{1-\rho}} \zeta_{u}^{\frac{1}{\alpha-1}}\left(\pi_{u} \zeta_{u}^{\frac{\alpha}{\alpha-1}}+\pi_{d} \zeta_{d}^{\frac{\alpha}{\alpha-1}}\right)^{\frac{\rho-\alpha}{\alpha(1-\rho)}}
$$

Substituting (3.5) and (3.6) into budget constraint (3.3), we get the following optimal consumption choice of the agent:

$$
\begin{aligned}
c_{0}^{* m} & =\frac{W}{1+\mathcal{A}}, \\
c_{u}^{* m} & =\beta^{\frac{\alpha}{\rho(1-\alpha)}} \mathcal{A}^{\frac{\rho-\alpha}{\rho(1-\alpha)}} \zeta_{u}^{\frac{1}{\alpha-1}} \frac{W}{1+\mathcal{A}}, \\
c_{d}^{* m} & =\beta^{\frac{\alpha}{\rho(1-\alpha)}} \mathcal{A}^{\frac{\rho-\alpha}{\rho(1-\alpha)}} \zeta_{d}^{\frac{1}{\alpha-1}} \frac{W}{1+\mathcal{A}},
\end{aligned}
$$

where

$$
\mathcal{A} \triangleq \beta^{\frac{1}{1-\rho}}\left(\pi_{u} \zeta_{u}^{\frac{\alpha}{\alpha-1}}+\pi_{d} \zeta_{d}^{\frac{\alpha}{\alpha-1}}\right)^{\frac{\rho(1-\alpha)}{\alpha(1-\rho)}}>0
$$


The agent's demand for consumptions in times and states is Marshallian and thus we have used superscript $m$ to stand for Marshallian demand.

The agent's optimal portfolio can be found by substituting (3.7) - (3.9) into the original budget constraints, (2.2) - (2.4).

$$
\begin{aligned}
\theta_{s}^{* m} & =\frac{\left(\zeta_{u}^{\frac{1}{\alpha-1}}-\zeta_{d}^{\frac{1}{\alpha-1}}\right) \beta^{\frac{\alpha}{\rho(1-\alpha)}} \mathcal{A}^{\frac{\rho-\alpha}{\rho(1-\alpha)}} W}{(u-d)(1+\mathcal{A})}, \\
\theta_{b}^{* m} & =\frac{\left(u \zeta_{d}^{\frac{1}{\alpha-1}}-d \zeta_{u}^{\frac{1}{\alpha-1}}\right) \beta^{\frac{\alpha}{\rho(1-\alpha)}} \mathcal{A}^{\frac{\rho-\alpha}{\rho(1-\alpha)}} W}{R(u-d)(1+\mathcal{A})} .
\end{aligned}
$$

If the risk premium on the stock is positive, i.e., $\zeta_{u}^{\frac{1}{\alpha-1}}>\zeta_{d}^{\frac{1}{\alpha-1}}$, then $\theta_{s}^{* m}>0$. From now on, we will assume that the risk premium is positive.

Value function $V(W)$ takes the following simple form

$$
V(W)=(1+\mathcal{A})^{\frac{1}{\rho}-1} W .
$$

We now consider the dual problem of the agent's optimization problem, the expenditure minimization problem. From (3.13) we derive the following expenditure function for utility level $\bar{u}$

$$
E(\bar{u})=(1+\mathcal{A})^{1-\frac{1}{\rho}} \bar{u} .
$$

And the agent's Hicksian demand for consumptions and assets can be found from the optimal choices of utility maximization problem by substituting $W$ by $E(\bar{u})$ in (3.14). The Hicksian demand for consumptions is given by the following

$$
\begin{aligned}
c_{0}^{* h} & =\frac{\bar{u}}{(1+\mathcal{A})^{\frac{1}{\rho}}}, \\
c_{u}^{* h} & =\beta^{\frac{\alpha}{\rho(1-\alpha)}} \mathcal{A}^{\frac{\rho-\alpha}{\rho(1-\alpha)}} \zeta_{u}^{\frac{1}{\alpha-1}} \frac{\bar{u}}{(1+\mathcal{A})^{\frac{1}{\rho}}}, \\
c_{d}^{* h} & =\beta^{\frac{\alpha}{\rho(1-\alpha)}} \mathcal{A}^{\frac{\rho-\alpha}{\rho(1-\alpha)}} \zeta_{d}^{\frac{1}{\alpha-1}} \frac{\bar{u}}{(1+\mathcal{A})^{\frac{1}{\rho}}},
\end{aligned}
$$

and the Hicksian demand for assets is given as

$$
\begin{aligned}
\theta_{s}^{* h} & =\frac{\left(\zeta_{u}^{\frac{1}{\alpha-1}}-\zeta_{d}^{\frac{1}{\alpha-1}}\right) \beta^{\frac{\alpha}{\rho(1-\alpha)}} \mathcal{A}^{\frac{\rho-\alpha}{\rho(1-\alpha)}} \bar{u}}{(u-d)(1+\mathcal{A})^{\frac{1}{\rho}}}, \\
\theta_{b}^{* h} & =\frac{\left(u \zeta_{d}^{\frac{1}{\alpha-1}}-d \zeta_{u}^{\frac{1}{\alpha-1}}\right) \beta^{\frac{\alpha}{\rho(1-\alpha)}} \mathcal{A}^{\frac{\rho-\alpha}{\rho(1-\alpha)}} \bar{u}}{R(u-d)(1+\mathcal{A})^{\frac{1}{\rho}}} .
\end{aligned}
$$


Superscript $h$ stands for Hicksian.

We will use (3.15) - (3.19) to easily derive Slutsky equations in the following section.

\section{The Substitution EfFect And the Income EFfect}

We now proceed to do comparative statics of the agent's consumption and portfolio choice. A well-known mathematical representation of the substitution effect and the income effect of a parameter change is the Slutsky equation. Cook [10] gave one-line proof of the Slutsky equation as follows. If we define the Marshallian demand function as

$$
M\left(p_{u}, p_{d}, W\right) \triangleq \arg \max _{c} U\left(c_{0}, c_{u}, c_{d}\right),
$$

and the Hicksian demand function as

$$
H\left(p_{u}, p_{d}, \bar{u}\right) \triangleq \arg \min _{c}\left\{c_{0}+p_{u} c_{u}+p_{d} c_{d}\right\},
$$

by definition, $H\left(p_{u}, p_{d}, \bar{u}\right)=M\left(p_{u}, p_{d}, E(\bar{u})\right)$. Thus Slutsky equation is

$$
\frac{\partial M}{\partial p_{i}}=\frac{\partial H}{\partial p_{i}}-\frac{\partial M}{\partial W} \cdot \frac{\partial E}{\partial p_{i}} .
$$

The first term on the right hand side in (4.1) represents the substitution effect and the second term represents the income effect.

4.1. The Effect of a Change in a State Price. We analyze the substitution effect and the income effect of state prices, $p_{u}$ and $p_{d}$ on consumption at time 0.

We decompose the effect of a change in $p_{u}$ on consumption at time 0 by deriving the Slutsky equation. Using (3.15) and (3.7), we derive the following Slutsky equation

$$
\begin{aligned}
\frac{\partial c_{0}^{* m}}{\partial p_{u}} & =\frac{\partial c_{0}^{* h}}{\partial p_{u}}-\frac{\partial c_{0}^{* m}}{\partial W} \frac{\partial E}{\partial p_{u}} \\
& =-\frac{\bar{u}}{\rho(1+\mathcal{A})^{\frac{1}{\rho}+1}} \frac{\partial \mathcal{A}}{\partial p_{u}}-\beta^{\frac{\alpha}{\rho(1-\alpha)}} \mathcal{A}^{\frac{\rho-\alpha}{\rho(1-\alpha)}} \zeta_{u}^{\frac{1}{\alpha-1}} \frac{W}{(1+\mathcal{A})^{2}}
\end{aligned}
$$

where

$$
\frac{\partial \mathcal{A}}{\partial p_{u}}=\frac{\rho}{\rho-1} \beta^{\frac{\alpha}{\rho(1-\alpha)}} \mathcal{A}^{\frac{\rho-\alpha}{\rho(1-\alpha)}} \zeta_{u}^{\frac{1}{\alpha-1}} .
$$

We see that $\rho$ determines the sign of (4.3).

$$
\left\{\begin{array}{l}
\frac{\partial \mathcal{A}}{\partial p_{u}}>0, \text { if } \rho<0, \\
\frac{\partial \mathcal{A}}{\partial p_{u}}<0, \text { if } 0<\rho<1 .
\end{array}\right.
$$

From (4.2), it is obvious that the income effect is negative. Since her purchasing power decreases due to an increased price, the effect is to reduce the agent's current consumption.

The sign of the substitution effect depends on the magnitude of EIS, $\sigma$. Since $\frac{1}{1-\rho}=\sigma$, EIS $<1$ if and only if $\rho<0$ and EIS $>1$ if and only if $0<\rho<1$. A large EIS (EIS $>1$ ) 
implies the agent is willing to substitute future consumption for current consumption, and thus the substitution effect of the change in the price of future consumption is positive. However, if EIS $<1$, the agent is not very willing to substitute future consumption for current consumption, the substitution effect is negative.

We can derive the total effect from (3.7).

$$
\frac{\partial c_{0}^{* m}}{\partial p_{u}}=-\frac{W}{(1+\mathcal{A})^{2}} \frac{\partial \mathcal{A}}{\partial p_{u}} .
$$

Therefore, if the agent's EIS is less than 1, the total effect is negative, since both the substitution effect and the income effect are negative. If the EIS is greater than 1, the total effect is positive and the substitution effect dominates the income effect.

\begin{tabular}{|c|c|c|c|}
\hline Case & $\begin{array}{c}\text { Sub. } \\
\text { effect }\end{array}$ & $\begin{array}{c}\text { Income } \\
\text { effect }\end{array}$ & $\begin{array}{c}\text { Total } \\
\text { effect }\end{array}$ \\
\hline EIS $<1$ & - & - & - \\
EIS $>1$ & + & - & + \\
\hline
\end{tabular}

TABLE 1. The signs of the substitution and income effects for $\partial c_{0}^{* m} / \partial p_{u}$

The effect of a change in $p_{d}$ on current consumption is similar to that of $p_{u}$.

Now we consider the effect of a change in one state price on optimal consumption in the other state, e.g., $\frac{\partial c_{u}^{* m}}{\partial p_{d}}$. The Slutsky equation is derived from (3.16) and (3.8).

$$
\begin{aligned}
\frac{\partial c_{u}^{* m}}{\partial p_{d}}= & \frac{\partial c_{u}^{* h}}{\partial p_{d}}-\frac{\partial c_{u}^{* m}}{\partial W} \frac{\partial E}{\partial p_{d}} \\
= & \frac{\rho-\alpha+(\rho-1) \mathcal{A}}{\rho(1-\alpha) \mathcal{A}(1+\mathcal{A})} \beta^{\frac{\alpha}{\rho(1-\alpha)}} \mathcal{A}^{\frac{\rho-\alpha}{\rho(1-\alpha)}} \zeta_{u}^{\frac{1}{\alpha-1}} \frac{\bar{u}}{(1+\mathcal{A})^{\frac{1}{\rho}}} \frac{\partial \mathcal{A}}{\partial p_{d}} \\
& -\beta^{\frac{2 \alpha}{\rho(1-\alpha)}} \mathcal{A}^{\frac{2(\rho-\alpha)}{\rho(1-\alpha)}} \zeta_{u}^{\frac{1}{\alpha-1}} \zeta_{d}^{\frac{1}{\alpha-1}} \frac{W}{(1+\mathcal{A})^{2}} .
\end{aligned}
$$

And it is simple to show that

$$
\left\{\begin{array}{l}
\frac{\partial \mathcal{A}}{\partial p_{d}}>0, \text { if } \rho<0 \\
\frac{\partial \mathcal{A}}{\partial p_{d}}<0, \text { if } 0<\rho<1 .
\end{array}\right.
$$

The term, $(\rho-\alpha+(\rho-1) \mathcal{A}) / \rho$, plays a key role determining the sign of the substitution effect. However, we are not able to determine the sign of the substitution effect unambiguously. Instead, we can claim that with a large relative risk aversion coefficient $\gamma$, the substitution effect is negative. That is, if the agent is highly risk-averse, regardless of the agent's EIS, the substitution effect is negative. On the other hand, if the agent is not very risk-averse and her 
EIS is large, as in (4.2), then she is willing to substitute consumption in state $d$ for consumption at time 0 , and the substitution effect on consumption in state $u$ can be negative.

The income effect is unambiguously negative; since the price has increased, the agent's purchasing power has declined.

We can derive the total effect from (3.8).

$$
\frac{\partial c_{u}^{* m}}{\partial p_{d}}=\frac{\rho-\alpha+\alpha(\rho-1) \mathcal{A}}{\rho(1-\alpha) \mathcal{A}(1+\mathcal{A})} \beta^{\frac{\alpha}{\rho(1-\alpha)}} \mathcal{A}^{\frac{\rho-\alpha}{\rho(1-\alpha)}} \zeta_{u}^{\frac{1}{\alpha-1}} \frac{W}{1+\mathcal{A}} \frac{\partial \mathcal{A}}{\partial p_{d}} .
$$

If $\rho<0$ (EIS $<1)$, since the sign of $(\rho-\alpha+\alpha(\rho-1) \mathcal{A}) / \rho$ is not determined, we are not able to determine the sign of the total effect. However, for a large risk aversion, it is negative. And when $0<\rho<1$ (EIS $>1),(\rho-\alpha+\alpha(\rho-1) \mathcal{A}) / \rho$ is positive and $\frac{\partial \mathcal{A}}{\partial p_{d}}$ is negative and thus the sign of the total effect is negative. When $p_{d}$ increases, the agent with an EIS $>1$ or with large risk aversion will reduce her consumption in state $u$.

\begin{tabular}{|c|c|c|c|}
\hline Case & $\begin{array}{c}\text { Sub. } \\
\text { effect }\end{array}$ & $\begin{array}{c}\text { Income } \\
\text { effect }\end{array}$ & $\begin{array}{c}\text { Total } \\
\text { effect }\end{array}$ \\
\hline EIS $<1$ & $+/-$ & - & $+/-$ \\
EIS $>1$ & $+/-$ & - & - \\
\hline
\end{tabular}

TABLE 2. The signs of the substitution and income effects for $\partial c_{u}^{* m} / \partial p_{d}$

A symmetric argument can be applied for $\frac{\partial c_{d}^{* m}}{\partial p_{u}}$.

We now analyze the effect of a change in a state price on the agent's portfolio choice. For investment in risky asset, the Slutsky equation is derived from (3.18) and (3.11) as follows

$$
\begin{aligned}
\frac{\partial \theta_{s}^{* m}}{\partial p_{u}}= & \frac{\partial \theta_{s}^{* h}}{\partial p_{u}}-\frac{\partial \theta_{s}^{* m}}{\partial W} \frac{\partial E}{\partial p_{u}} \\
= & \frac{\left(\zeta_{u}^{\frac{1}{\alpha-1}}-\zeta_{d}^{\frac{1}{\alpha-1}}\right) \beta^{\frac{\alpha}{\rho(1-\alpha)}} \mathcal{A}^{\frac{\rho-\alpha}{\rho(1-\alpha)}} \bar{u}}{(u-d)(1+\mathcal{A})^{\frac{1}{\rho}}}\left(\frac{\rho-\alpha+(\rho-1) \mathcal{A}}{\rho(1-\alpha) \mathcal{A}(1+\mathcal{A})}\right) \frac{\partial \mathcal{A}}{\partial p_{u}} \\
& -\frac{\beta^{\frac{\alpha}{\rho(1-\alpha)}} \mathcal{A}^{\frac{\rho-\alpha}{\rho(1-\alpha)}} \zeta_{u}^{\frac{1}{\alpha-1}} \bar{u}}{p_{u}(1-\alpha)(u-d)(1+\mathcal{A})^{\frac{1}{\rho}}}-\frac{\left(\zeta_{u}^{\frac{1}{\alpha-1}}-\zeta_{d}^{\frac{1}{\alpha-1}}\right) \beta^{\frac{2 \alpha}{\rho(1-\alpha)}} \mathcal{A}^{\frac{2(\rho-\alpha)}{\rho(1-\alpha)}} \zeta_{u}^{\frac{1}{\alpha-1}} W}{(u-d)(1+\mathcal{A})^{2}}
\end{aligned}
$$

The first two terms exhibit the substitution effect. The second term is unambiguously negative, but the sign of the first term is not determined due to term, $(\rho-\alpha+(\rho-1) \mathcal{A}) / \rho$. However, if $0<\rho<1$ and $\gamma$ is large, the substitution effect is negative. This is because when the price of a future consumption increases, an agent with a large EIS will increase current consumption and reduce savings. And the income effect is negative since the agent's purchasing power has declined. 
We can derive the total effect of $\frac{\partial \theta_{s}^{* m}}{\partial p_{u}}$ from (3.11).

$$
\begin{aligned}
\frac{\partial \theta_{s}^{* m}}{\partial p_{u}}= & \frac{\left(\zeta_{u}^{\frac{1}{\alpha-1}}-\zeta_{d}^{\frac{1}{\alpha-1}}\right) \beta^{\frac{\alpha}{\rho(1-\alpha)}} \mathcal{A}^{\frac{\rho-\alpha}{\rho(1-\alpha)} W}}{(u-d)(1+\mathcal{A})}\left(\frac{\rho-\alpha+\alpha(\rho-1) \mathcal{A}}{\rho(1-\alpha) \mathcal{A}(1+\mathcal{A})}\right) \frac{\partial \mathcal{A}}{\partial p_{u}} \\
& -\frac{\beta^{\frac{\alpha}{\rho(1-\alpha)}} \mathcal{A}^{\frac{\rho-\alpha}{\rho(1-\alpha)}} \zeta_{u}^{\frac{1}{\alpha-1}} W}{p_{u}(1-\alpha)(u-d)(1+\mathcal{A})} .
\end{aligned}
$$

If $\rho<0$ (EIS $<1$ ), the sign of $(\rho-\alpha+\alpha(\rho-1) \mathcal{A}) / \rho$ is not determined and thus the sign of the total effect is ambiguous. Again, with a large $\gamma$, it is negative. And if $0<\rho<1$ (EIS $>1$ ), the total effect is negative. Therefore, when the price of future consumption increases, an agent with an EIS $>1$ or with large risk aversion will reduce her investment in the risky asset.

\begin{tabular}{|c|c|c|c|}
\hline Case & $\begin{array}{c}\text { Sub. } \\
\text { effect }\end{array}$ & $\begin{array}{c}\text { Income } \\
\text { effect }\end{array}$ & $\begin{array}{c}\text { Total } \\
\text { effect }\end{array}$ \\
\hline EIS $<1$ & $+/-$ & - & $+/-$ \\
EIS $>1$ & $+/-$ & - & - \\
\hline
\end{tabular}

TABLE 3. The signs of the substitution and income effects for $\partial \theta_{s}^{* m} / \partial p_{u}$

The Slutsky equation for investment in the bond is derived from (3.19) and (3.12) as follows

$$
\begin{aligned}
\frac{\partial \theta_{b}^{* m}}{\partial p_{u}}= & \frac{\partial \theta_{b}^{* h}}{\partial p_{u}}-\frac{\partial \theta_{b}^{* m}}{\partial W} \frac{\partial E}{\partial p_{u}} \\
= & \frac{\left(u \zeta_{d}^{\frac{1}{\alpha-1}}-d \zeta_{u}^{\frac{1}{\alpha-1}}\right) \beta^{\frac{\alpha}{\rho(1-\alpha)}} \mathcal{A}^{\frac{\rho-\alpha}{\rho(1-\alpha)}} \bar{u}}{R(u-d)(1+\mathcal{A})^{\frac{1}{\rho}}}\left(\frac{\rho-\alpha+(\rho-1) \mathcal{A}}{\rho(1-\alpha) \mathcal{A}(1+\mathcal{A})}\right) \frac{\partial \mathcal{A}}{\partial p_{u}} \\
& +\frac{d \beta^{\frac{\alpha}{\rho(1-\alpha)}} \mathcal{A}^{\frac{\rho-\alpha}{\rho(1-\alpha)}} \zeta_{u}^{\frac{1}{\alpha-1}} \bar{u}}{p_{u}(1-\alpha) R(u-d)(1+\mathcal{A})^{\frac{1}{\rho}}}-\frac{\left(u \zeta_{d}^{\frac{1}{\alpha-1}}-d \zeta_{u}^{\frac{1}{\alpha-1}}\right) \beta^{\frac{2 \alpha}{\rho(1-\alpha)}} \mathcal{A}^{\frac{2(\rho-\alpha)}{\rho(1-\alpha)}} \zeta_{u}^{\frac{1}{\alpha-1}} W}{R(u-d)(1+\mathcal{A})^{2}} .
\end{aligned}
$$

The first two terms show the substitution effect and the signs of the substitution effect and the income effect are ambiguous, since the sign of term $\left(u \zeta_{d}^{\frac{1}{\alpha-1}}-d \zeta_{u}^{\frac{1}{\alpha-1}}\right)$ is not determined.

And the total effect can be derived from (3.12) as follows

$$
\begin{aligned}
\frac{\partial \theta_{b}^{* m}}{\partial p_{u}}= & \frac{\left(u \zeta_{d}^{\frac{1}{\alpha-1}}-d \zeta_{u}^{\frac{1}{\alpha-1}}\right) \beta^{\frac{\alpha}{\rho(1-\alpha)}} \mathcal{A}^{\frac{\rho-\alpha}{\rho(1-\alpha)}} W}{R(u-d)(1+\mathcal{A})}\left(\frac{\rho-\alpha+\alpha(\rho-1) \mathcal{A}}{\rho(1-\alpha) \mathcal{A}(1+\mathcal{A})}\right) \frac{\partial \mathcal{A}}{\partial p_{u}} \\
& +\frac{d \beta^{\frac{\alpha}{\rho(1-\alpha)}} \mathcal{A}^{\frac{\rho-\alpha}{\rho(1-\alpha)}} \zeta_{u}^{\frac{1}{\alpha-1}} W}{p_{u}(1-\alpha) R(u-d)(1+\mathcal{A})} .
\end{aligned}
$$


The sign of the total effect is also is ambiguous.

\begin{tabular}{|c|c|c|c|}
\hline Case & $\begin{array}{c}\text { Sub. } \\
\text { effect }\end{array}$ & $\begin{array}{c}\text { Income } \\
\text { effect }\end{array}$ & $\begin{array}{c}\text { Total } \\
\text { effect }\end{array}$ \\
\hline EIS $<1$ & $+/-$ & $+/-$ & $+/-$ \\
EIS $>1$ & $+/-$ & $+/-$ & $+/-$ \\
\hline
\end{tabular}

TABLE 4. The signs of effect of $\partial \theta_{b}^{* m} / \partial p_{u}$

The effect of a change in $p_{d}$ on portfolio choice is similar to that of $p_{u}$.

4.2. The Effect of a Change in the Interest Rate. Now let us consider the effect of a change in the interest rate. We first make the following observation which can be derived from (2.6) and (2.7):

$$
\begin{aligned}
& \frac{\partial p_{u}}{\partial R}=-\frac{d}{(u-d) R^{2}}<0, \\
& \frac{\partial p_{d}}{\partial R}=-\frac{u}{(u-d) R^{2}}<0 .
\end{aligned}
$$

When the interest rate increases, both state prices decline so that the equality, $p_{u}+p_{d}=1 / R$, still holds.

We can derive the following Slutsky equation for the effect of a change in the interest rate on consumption at time 0 :

$$
\begin{aligned}
\frac{\partial c_{0}^{* m}}{\partial R} & =\frac{\partial c_{0}^{* h}}{\partial R}-\frac{\partial c_{0}^{* m}}{\partial W} \frac{\partial E}{\partial R} \\
& =-\frac{\bar{u}}{\rho(1+\mathcal{A})^{\frac{1}{\rho}+1}} \frac{\partial \mathcal{A}}{\partial R}-\frac{1}{1+\mathcal{A}}\left(\frac{\partial p_{u}}{\partial R} c_{u}^{* m}+\frac{\partial p_{d}}{\partial R} c_{d}^{* m}\right) \\
& =-\frac{\bar{u}}{\rho(1+\mathcal{A})^{\frac{1}{\rho}+1}} \frac{\partial \mathcal{A}}{\partial R}-\beta^{\frac{\alpha}{\rho(1-\alpha)}} \mathcal{A}^{\frac{\rho-\alpha}{\rho(1-\alpha)}} \frac{W}{(1+\mathcal{A})^{2}}\left(\zeta_{u}^{\frac{1}{\alpha-1}} \frac{\partial p_{u}}{\partial R}+\zeta_{d}^{\frac{1}{\alpha-1}} \frac{\partial p_{d}}{\partial R}\right)(4.8)
\end{aligned}
$$

where

$$
\frac{\partial \mathcal{A}}{\partial R}=\frac{\rho}{\rho-1} \beta^{\frac{\alpha}{\rho(1-\alpha)}} \mathcal{A}^{\frac{\rho-\alpha}{\rho(1-\alpha)}}\left(\zeta_{u}^{\frac{1}{\alpha-1}} \frac{\partial p_{u}}{\partial R}+\zeta_{d}^{\frac{1}{\alpha-1}} \frac{\partial p_{d}}{\partial R}\right) .
$$

We have applied the envelop theorem to obtain the second equality. And we know that

$$
\left\{\begin{array}{l}
\frac{\partial \mathcal{A}}{\partial R}<0, \text { if } \rho<0 \\
\frac{\partial \mathcal{A}}{\partial R}>0, \text { if } 0<\rho<1 .
\end{array}\right.
$$

In (4.8) the substitution effect is always negative regardless of whether the EIS is smaller than or larger than 1. If the interest rate increases, the price of future consumption decreases and 
the agent will increase future consumption and reduce current consumption. And the income effect is positive, since the agent's purchasing power increases with an increase in the interest rate.

From (3.7) we derive the following total effect:

$$
\frac{\partial c_{0}^{* m}}{\partial R}=-\frac{W}{(1+\mathcal{A})^{2}} \frac{\partial \mathcal{A}}{\partial R} .
$$

The EIS determines the sign of the total effect. When the EIS is less than 1, the total effect is positive. Since the EIS is small, the income effect dominates the substitution effect. And if when EIS is larger than 1, the total effect is negative and thus the substitution effect dominates the income effect. Therefore, when the interest rate increases, an agent with an EIS $<1$ will increase her current consumption and an agent with an EIS $>1$ will reduce her current consumption.

\begin{tabular}{|c|c|c|c|}
\hline Case & $\begin{array}{c}\text { Sub. } \\
\text { effect }\end{array}$ & $\begin{array}{c}\text { Income } \\
\text { effect }\end{array}$ & $\begin{array}{c}\text { Total } \\
\text { effect }\end{array}$ \\
\hline EIS $<1$ & - & + & + \\
EIS $>1$ & - & + & - \\
\hline
\end{tabular}

TABLE 5. The signs of the substitution and income effects for $\partial c_{0}^{* m} / \partial R$

We can now derive the following Slutsky equation for $c_{u}^{* m}$ from (3.16) and (3.8):

$$
\begin{aligned}
\frac{\partial c_{u}^{* m}}{\partial R}= & \frac{\partial c_{u}^{* h}}{\partial R}-\frac{\partial c_{u}^{* m}}{\partial W} \frac{\partial E}{\partial R} \\
= & \beta^{\frac{\alpha}{\rho(1-\alpha)}} \mathcal{A}^{\frac{\rho-\alpha}{\rho(1-\alpha)}} \zeta_{u}^{\frac{1}{\alpha-1}} \frac{\bar{u}}{(1+\mathcal{A})^{\frac{1}{\rho}}}\left(\frac{\rho-\alpha+(\rho-1) \mathcal{A}}{\rho(1-\alpha) \mathcal{A}(1+\mathcal{A})} \frac{\partial \mathcal{A}}{\partial R}-\frac{1}{p_{u}(1-\alpha)} \frac{\partial p_{u}}{\partial R}\right) \\
& -\beta^{\frac{2 \alpha}{\rho(1-\alpha)}} \mathcal{A}^{\frac{2(\rho-\alpha)}{\rho(1-\alpha)}} \frac{W}{(1+\mathcal{A})^{2}}\left(\frac{\partial p_{u}}{\partial R} \zeta_{u}^{\frac{1}{\alpha-1}}+\frac{\partial p_{d}}{\partial R} \zeta_{d}^{\frac{1}{\alpha-1}}\right) .
\end{aligned}
$$

It is clear that the income effect is positive with an increased purchasing power. For the substitution effect, the second term, $-\frac{1}{p_{u}(1-\alpha)} \frac{\partial p_{u}}{\partial R}$, in the parenthesis of the first term is positive. Due to term $(\rho-\alpha+(\rho-1) \mathcal{A}) / \rho$, however, the sign of the substitution effect is ambiguous. But when the agent's risk aversion is large, the substitution effect is positive.

We can derive the total effect from (3.8).

$$
\frac{\partial c_{u}^{* m}}{\partial R}=\beta^{\frac{\alpha}{\rho(1-\alpha)}} \mathcal{A}^{\frac{\rho-\alpha}{\rho(1-\alpha)}} \zeta_{u}^{\frac{1}{\alpha-1}} \frac{W}{1+\mathcal{A}}\left(\frac{\rho-\alpha+\alpha(\rho-1) \mathcal{A}}{\rho(1-\alpha) \mathcal{A}(1+\mathcal{A})} \frac{\partial \mathcal{A}}{\partial R}-\frac{1}{p_{u}(1-\alpha)} \frac{\partial p_{u}}{\partial R}\right) .
$$

If $\rho<0$ (EIS $<1$ ) the sign of the total effect is ambiguous. With large risk aversion, it is positive. If $0<\rho<1$ (EIS $>1$ ), the total effect is positive. Therefore, when the interest rate increases, the agent with a large EIS or with large risk aversion will increase her consumption in state $u$. 


\begin{tabular}{|c|c|c|c|}
\hline Case & $\begin{array}{c}\text { Sub. } \\
\text { effect }\end{array}$ & $\begin{array}{c}\text { Income } \\
\text { effect }\end{array}$ & $\begin{array}{c}\text { Total } \\
\text { effect }\end{array}$ \\
\hline EIS $<1$ & - & + & $+/-$ \\
EIS $>1$ & - & + & + \\
\hline
\end{tabular}

TABLE 6. The signs of the substitution and income effects for $\partial c_{u}^{* m} / \partial R$

We now analyze the effect of a change in the interest rate on the agent's portfolio choice. We can derive the following Slutsky equation

$$
\begin{aligned}
\frac{\partial \theta_{s}^{* m}}{\partial R}= & \frac{\partial \theta_{s}^{* h}}{\partial R}-\frac{\partial \theta_{s}^{* m}}{\partial W} \frac{\partial E}{\partial R} \\
= & \frac{\left(\zeta_{u}^{\frac{1}{\alpha-1}}-\zeta_{d}^{\frac{1}{\alpha-1}}\right) \beta^{\frac{\alpha}{\rho(1-\alpha)}} \mathcal{A}^{\frac{\rho-\alpha}{\rho(1-\alpha)}} \bar{u}}{(u-d)(1+\mathcal{A})^{\frac{1}{\rho}}}\left(\frac{\rho-\alpha+(\rho-1) \mathcal{A}}{\rho(1-\alpha) \mathcal{A}(1+\mathcal{A})}\right) \frac{\partial \mathcal{A}}{\partial R} \\
& +\frac{\beta^{\frac{\alpha}{\rho(1-\alpha)}} \mathcal{A}^{\frac{\rho-\alpha}{\rho(1-\alpha)}} \bar{u}}{(u-d)(1+\mathcal{A})^{\frac{1}{\rho}}}\left(\frac{\zeta_{d}^{\frac{1}{\alpha-1}}}{p_{d}(1-\alpha)} \frac{\partial p_{d}}{\partial R}-\frac{\zeta_{u}^{\frac{1}{\alpha-1}}}{p_{u}(1-\alpha)} \frac{\partial p_{u}}{\partial R}\right) \\
& -\frac{\left(\zeta_{u}^{\frac{1}{\alpha-1}}-\zeta_{d}^{\frac{1}{\alpha-1}}\right) \beta^{\frac{2 \alpha}{\rho(1-\alpha)}} \mathcal{A}^{\frac{2(\rho-\alpha)}{\rho(1-\alpha)}} W}{(u-d)(1+\mathcal{A})^{2}}\left(\zeta_{u}^{\frac{1}{\alpha-1}} \frac{\partial p_{u}}{\partial R}+\zeta_{d}^{\frac{1}{\alpha-1}} \frac{\partial p_{d}}{\partial R}\right)
\end{aligned}
$$

The first two terms exhibit the substitution effect and the signs of both terms are ambiguous. But the income effect is unambiguously positive, since the agent's purchasing power increases with an increase in the interest rate.

We derive the total effect from (3.11).

$$
\begin{aligned}
\frac{\partial \theta_{s}^{* m}}{\partial R}= & \frac{\left(\zeta_{u}^{\frac{1}{\alpha-1}}-\zeta_{d}^{\frac{1}{\alpha-1}}\right) \beta^{\frac{\alpha}{\rho(1-\alpha)}} \mathcal{A}^{\frac{\rho-\alpha}{\rho(1-\alpha)}} W}{(u-d)(1+\mathcal{A})}\left(\frac{\rho-\alpha+\alpha(\rho-1) \mathcal{A}}{\rho(1-\alpha) \mathcal{A}(1+\mathcal{A})}\right) \frac{\partial \mathcal{A}}{\partial R} \\
& +\frac{\beta^{\frac{\alpha}{\rho(1-\alpha)}} \mathcal{A}^{\frac{\rho-\alpha}{\rho(1-\alpha)}} W}{(u-d)(1+\mathcal{A})}\left(\frac{\zeta_{d}^{\frac{1}{\alpha-1}}}{p_{d}(1-\alpha)} \frac{\partial p_{d}}{\partial R}-\frac{\zeta_{u}^{\frac{1}{\alpha-1}}}{p_{u}(1-\alpha)} \frac{\partial p_{u}}{\partial R}\right) .
\end{aligned}
$$

The sign of the total effect is ambiguous in both cases, $\rho<0$ and $0<\rho<1$. 


\begin{tabular}{|c|c|c|c|}
\hline Case & $\begin{array}{c}\text { Sub. } \\
\text { effect }\end{array}$ & $\begin{array}{c}\text { Income } \\
\text { effect }\end{array}$ & $\begin{array}{c}\text { Total } \\
\text { effect }\end{array}$ \\
\hline EIS $<1$ & $+/-$ & + & $+/-$ \\
EIS $>1$ & $+/-$ & + & $+/-$ \\
\hline
\end{tabular}

TABLE 7. The signs of the substitution and income effects for $\partial \theta_{s}^{* m} / \partial R$

For investment in the risk-free bond, we can derive the following Slutsky equation from (3.19) and (3.12):

$$
\begin{aligned}
\frac{\partial \theta_{b}^{* m}}{\partial R}= & \frac{\partial \theta_{b}^{* h}}{\partial R}-\frac{\partial \theta_{b}^{* m}}{\partial W} \frac{\partial E}{\partial R} \\
= & \frac{\left(u \zeta_{d}^{\frac{1}{\alpha-1}}-d \zeta_{u}^{\frac{1}{\alpha-1}}\right) \beta^{\frac{\alpha}{\rho(1-\alpha)}} \mathcal{A}^{\frac{\rho-\alpha}{\rho(1-\alpha)}} \bar{u}}{R(u-d)(1+\mathcal{A})^{\frac{1}{\rho}}}\left[\left(\frac{\rho-\alpha+(\rho-1) \mathcal{A}}{\rho(1-\alpha) \mathcal{A}(1+\mathcal{A})}\right) \frac{\partial \mathcal{A}}{\partial R}+\frac{1}{R}\right] \\
& +\frac{\beta^{\frac{\alpha}{\rho(1-\alpha)}} \mathcal{A}^{\frac{\rho-\alpha}{\rho(1-\alpha)}} \bar{u}}{R(u-d)(1+\mathcal{A})^{\frac{1}{\rho}}}\left(\frac{d \zeta_{u}^{\frac{1}{\alpha-1}}}{p_{u}(1-\alpha)} \frac{\partial p_{u}}{\partial R}-\frac{u \zeta_{d}^{\frac{1}{\alpha-1}}}{p_{d}(1-\alpha)} \frac{\partial p_{d}}{\partial R}\right) \\
& -\frac{\left(u \zeta_{d}^{\frac{1}{\alpha-1}}-d \zeta_{u}^{\frac{1}{\alpha-1}}\right) \beta^{\frac{2 \alpha}{\rho(1-\alpha)}} \mathcal{A}^{\frac{2(\rho-\alpha)}{\rho(1-\alpha)}} W}{R(u-d)(1+\mathcal{A})^{2}}\left(\zeta_{u}^{\frac{1}{\alpha-1}} \frac{\partial p_{u}}{\partial R}+\zeta_{d}^{\frac{1}{\alpha-1}} \frac{\partial p_{d}}{\partial R}\right)
\end{aligned}
$$

The first two terms show the substitution effect, and we are not able to determine the sign of each term. And also the sign of the income effect is not determined.

The indeterminacy of the sign of the substitution effect in (4.11) can be explained by Dalal [6]'s intuitive argument. His model considers only portfolio choice but the argument is still applicable here. The substitution effect is measured in a situation where the agent minimizes expenditure to keep utility level constant. Suppose that the interest rate increases but the risk premium stays still positive. Suppose also that the agent is not very risk averse and thus reduces her bond investment by a small amount without changing her stock investment in an attempt to keep her expected utility unchanged and take advantage of the risk premium. Since the overall risk of return has decreased, however, her action would probably raise her expected utility. To keep the utility level constant she might need to reduce her bond holding further. In the other case where the agent is severely risk-averse, the agent will increase her bond investment and reduce her stock investment and thus lowers the expected return and risk of her portfolio so that her expected utility is kept constant.

Though Dalal's argument is for portfolio choice, we can apply it to consumption choice as well. For example, (4.10) shows a similar behavior. If the agent is heavily risk-averse, i.e., $\gamma$ is large, $\rho-\alpha+(\rho-1) \mathcal{A}$ is positive and thus the substitution effect becomes positive in both cases EIS $<1$ and when EIS $>1$. 
The total effect of the interest rate change on the agent's investment in the risk-free bond is given as

$$
\begin{aligned}
\frac{\partial \theta_{b}^{* m}}{\partial R}= & \frac{\left(u \zeta_{d}^{\frac{1}{\alpha-1}}-d \zeta_{u}^{\frac{1}{\alpha-1}}\right) \beta^{\frac{\alpha}{\rho(1-\alpha)}} \mathcal{A}^{\frac{\rho-\alpha}{\rho(1-\alpha)}} W}{R(u-d)(1+\mathcal{A})}\left[\left(\frac{\rho-\alpha+\alpha(\rho-1) \mathcal{A}}{\rho(1-\alpha) \mathcal{A}(1+\mathcal{A})}\right) \frac{\partial \mathcal{A}}{\partial R}+\frac{1}{R}\right] \\
& +\frac{\beta^{\frac{\alpha}{\rho(1-\alpha)}} \mathcal{A}^{\frac{\rho-\alpha}{\rho(1-\alpha)}} W}{R(u-d)(1+\mathcal{A})}\left(\frac{d \zeta_{u}^{\frac{1}{\alpha-1}}}{p_{u}(1-\alpha)} \frac{\partial p_{u}}{\partial R}-\frac{u \zeta_{d}^{\frac{1}{\alpha-1}}}{p_{d}(1-\alpha)} \frac{\partial p_{d}}{\partial R}\right)
\end{aligned}
$$

The sign of the total effect is ambiguous.

\begin{tabular}{|c|c|c|c|}
\hline Case & $\begin{array}{c}\text { Sub. } \\
\text { effect }\end{array}$ & $\begin{array}{c}\text { Income } \\
\text { effect }\end{array}$ & $\begin{array}{c}\text { Total } \\
\text { effect }\end{array}$ \\
\hline EIS $<1$ & $+/-$ & $+/-$ & $+/-$ \\
EIS $>1$ & $+/-$ & $+/-$ & $+/-$ \\
\hline
\end{tabular}

TABLE 8. The signs of the substitution and income effects for $\partial \theta_{b}^{* m} / \partial R$

4.3. The Effect of a Change in the Return on the Stock. We now analyze the effect of a change in the return on the stock. When the return changes, it changes the state prices. From (2.6) and (2.7) we observe

$$
\frac{\partial p_{d}}{\partial u}=-\frac{\partial p_{u}}{\partial u}=\frac{R-d}{(u-d)^{2} R} \triangleq \frac{\partial p}{\partial u}>0 .
$$

We derive the following Slutsky equation for $\frac{\partial c_{0}^{* m}}{\partial u}$ from (3.15) and (3.7):

$$
\begin{aligned}
\frac{\partial c_{0}^{* m}}{\partial u} & =\frac{\partial c_{0}^{* h}}{\partial u}-\frac{\partial c_{0}^{* m}}{\partial W} \frac{\partial E}{\partial u} \\
& =-\frac{\bar{u}}{\rho(1+\mathcal{A})^{\frac{1}{\rho}+1}} \frac{\partial \mathcal{A}}{\partial u}+\beta^{\frac{\alpha}{\rho(1-\alpha)}} \mathcal{A}^{\frac{\rho-\alpha}{\rho(1-\alpha)}}\left(\zeta_{u}^{\frac{1}{\alpha-1}}-\zeta_{d}^{\frac{1}{\alpha-1}}\right) \frac{W}{(1+\mathcal{A})^{2}} \frac{\partial p}{\partial u},
\end{aligned}
$$

where

It is simple to show that

$$
\frac{\partial \mathcal{A}}{\partial u}=\frac{\rho}{\rho-1} \beta^{\frac{\alpha}{\rho(1-\alpha)}} \mathcal{A}^{\frac{\rho-\alpha}{\rho(1-\alpha)}}\left(\zeta_{d}^{\frac{1}{\alpha-1}}-\zeta_{u}^{\frac{1}{\alpha-1}}\right) \frac{\partial p}{\partial u} .
$$

$$
\left\{\begin{array}{l}
\frac{\partial \mathcal{A}}{\partial u}<0, \text { if } \rho<0 \\
\frac{\partial \mathcal{A}}{\partial u}>0, \text { if } 0<\rho<1 .
\end{array}\right.
$$

Therefore, the substitution effect is negative and the income effect is positive. When $u$ increases, the expected return on the stock increases and the agent will increase her investment 
in it and has incentive to reduce current consumption. Since the consumption in state $u$ is now cheaper, her purchasing power increases.

Let us now compute the total effect from (3.7). Since

$$
\frac{\partial c_{0}^{* m}}{\partial u}=-\frac{W}{(1+\mathcal{A})^{2}} \frac{\partial \mathcal{A}}{\partial u}
$$

If $\rho<0$ (EIS $<1$ ), the income effect dominates the substitution effect and thus the total effect is positive. If $0<\rho<1$ (EIS $>1$ ), the substitution effect dominates the income effect and the total effect is negative. Thus, when $u$ increases, an agent with an EIS $<1$ will increase her consumption at time 0 due to increased purchasing power, she is not much willing to substitute current consumption for future consumption. An agent with an EIS $>1$ will reduce her current consumption due to the lower price of consumption in state $u$.

\begin{tabular}{|c|c|c|c|}
\hline Case & $\begin{array}{c}\text { Sub. } \\
\text { effect }\end{array}$ & $\begin{array}{c}\text { Income } \\
\text { effect }\end{array}$ & $\begin{array}{c}\text { Total } \\
\text { effect }\end{array}$ \\
\hline EIS $<1$ & - & + & + \\
EIS $>1$ & - & + & - \\
\hline
\end{tabular}

TABLE 9. The signs of the substitution and income effects for $\partial c_{0}^{* m} / \partial u$

A similar argument can be applied to a change of $d$.

We now consider the effect of a change in the stock's return on future consumption. Here we will study the effect of a change in $d$. The Slutsky equation for $\frac{\partial c_{u}^{* m}}{\partial d}$ can derived as follows

$$
\begin{aligned}
\frac{\partial c_{u}^{* m}}{\partial d}= & \frac{\partial c_{u}^{* h}}{\partial d}-\frac{\partial c_{u}^{* m}}{\partial W} \frac{\partial E}{\partial d} \\
= & \beta^{\frac{\alpha}{\rho(1-\alpha)}} \mathcal{A}^{\frac{\rho-\alpha}{\rho(1-\alpha)}} \zeta_{u}^{\frac{1}{\alpha-1}} \frac{\bar{u}}{(1+\mathcal{A})^{\frac{1}{\rho}}}\left(\frac{\rho-\alpha+(\rho-1) \mathcal{A}}{\rho(1-\alpha) \mathcal{A}(1+\mathcal{A})} \frac{\partial \mathcal{A}}{\partial d}+\frac{1}{p_{u}(1-\alpha)} \frac{\partial p}{\partial d}\right) \\
& +\beta^{\frac{2 \alpha}{\rho(1-\alpha)}} \mathcal{A}^{\frac{2(\rho-\alpha)}{\rho(1-\alpha)}} \zeta_{u}^{\frac{1}{\alpha-1}}\left(\zeta_{u}^{\frac{1}{\alpha-1}}-\zeta_{d}^{\frac{1}{\alpha-1}}\right) \frac{W}{(1+\mathcal{A})^{2}} \frac{\partial p}{\partial d}
\end{aligned}
$$

where

$$
\frac{\partial p_{d}}{\partial d}=-\frac{\partial p_{u}}{\partial d}=\frac{u-R}{(u-d)^{2} R} \triangleq \frac{\partial p}{\partial d}>0
$$

We observe that the term, $(\rho-\alpha+(\rho-1) \mathcal{A}) / \rho$, determines the sign of the substitution effect, and thus we are not able to easily determine the sign of the substitution effect. However, with a large $\gamma$ it is positive. It is because an increase in return $d$ results in a decrease in $p_{u}$ and thus the agent will increase consumption in state $u$. And the income effect is positive, since the return on the risky asset has increased and the agent has a larger purchasing power. 
We can derive the total effect from (3.8).

$$
\frac{\partial c_{u}^{* m}}{\partial d}=\beta^{\frac{\alpha}{\rho(1-\alpha)}} \mathcal{A}^{\frac{\rho-\alpha}{\rho(1-\alpha)}} \zeta_{u}^{\frac{1}{\alpha-1}}\left(\frac{\rho-\alpha+\alpha(\rho-1) \mathcal{A}}{\rho(1-\alpha) \mathcal{A}(1+\mathcal{A})} \frac{\partial \mathcal{A}}{\partial u}+\frac{1}{p_{u}(1-\alpha)} \frac{\partial p}{\partial d}\right) \frac{W}{1+\mathcal{A}} .
$$

When $\rho<0$ (EIS $<1$ ), the sign of the term, $\rho-\alpha+\alpha(\rho-1) \mathcal{A}$, is ambiguous. With large risk aversion, however, the total effect is positive. And when $0<\rho<1$ (EIS $>1$ ), the total effect is clearly positive. It is interesting that when $d$ increases, the agent increases consumption in state $u$. Therefore, when $d$ increases, an agent with a large EIS or with large risk aversion will increase consumption in state $u$.

\begin{tabular}{|c|c|c|c|}
\hline Case & $\begin{array}{c}\text { Sub. } \\
\text { effect }\end{array}$ & $\begin{array}{c}\text { Income } \\
\text { effect }\end{array}$ & $\begin{array}{c}\text { Total } \\
\text { effect }\end{array}$ \\
\hline EIS $<1$ & $+/-$ & + & $+/-$ \\
EIS $>1$ & $+/-$ & + & + \\
\hline
\end{tabular}

TABLE 10. The signs of the substitution and income effects for $\partial c_{u}^{* m} / \partial d$

Now we analyze the effect of a change in the stock's return on the agent's portfolio choice. For investment in risky asset, the following Slutsky equation can be derived from (3.18) and (3.11):

$$
\begin{aligned}
\frac{\partial \theta_{s}^{* m}}{\partial u}= & \frac{\partial \theta_{s}^{* h}}{\partial u}-\frac{\partial \theta_{s}^{* m}}{\partial W} \frac{\partial E}{\partial u} \\
= & \frac{\left(\zeta_{u}^{\frac{1}{\alpha-1}}-\zeta_{d}^{\frac{1}{\alpha-1}}\right) \beta^{\frac{\alpha}{\rho(1-\alpha)}} \mathcal{A}^{\frac{\rho-\alpha}{\rho(1-\alpha)}} \bar{u}}{(u-d)(1+\mathcal{A})^{\frac{1}{\rho}}}\left[\left(\frac{\rho-\alpha+(\rho-1) \mathcal{A}}{\rho(1-\alpha) \mathcal{A}(1+\mathcal{A})}\right) \frac{\partial \mathcal{A}}{\partial u}+\frac{1}{u-d}\right] \\
& +\frac{\beta^{\frac{\alpha}{\rho(1-\alpha)}} \mathcal{A}^{\frac{\rho-\alpha}{\rho(1-\alpha)}} \bar{u}}{(1-\alpha)(u-d)(1+\mathcal{A})^{\frac{1}{\rho}}}\left(\frac{\zeta_{d}^{\frac{1}{\alpha-1}}}{p_{d}}+\frac{\zeta_{u}^{\frac{1}{\alpha-1}}}{p_{u}}\right) \frac{\partial p}{\partial u} \\
& +\frac{\left(\zeta_{u}^{\frac{1}{\alpha-1}}-\zeta_{d}^{\frac{1}{\alpha-1}}\right)^{2} \beta^{\frac{2 \alpha}{\rho(1-\alpha)}} \mathcal{A}^{\frac{2(\rho-\alpha)}{\rho(1-\alpha)}} W}{(u-d)(1+\mathcal{A})^{2}} \frac{\partial p}{\partial u} .
\end{aligned}
$$

The first two terms show the substitution effect. The second term is positive, but the sign of first term is not determined, and thus, the sign of substitution effect is ambiguous. However, with large risk aversion, it is positive. The income effect is always positive. 
The total effect on the investment in the stock can be derived as follows

$$
\begin{aligned}
\frac{\partial \theta_{s}^{* m}}{\partial u}= & \frac{\left(\zeta_{u}^{\frac{1}{\alpha-1}}-\zeta_{d}^{\frac{1}{\alpha-1}}\right) \beta^{\frac{\alpha}{\rho(1-\alpha)}} \mathcal{A}^{\frac{\rho-\alpha}{\rho(1-\alpha)}} W}{(u-d)(1+\mathcal{A})}\left[\left(\frac{\rho-\alpha+\alpha(\rho-1) \mathcal{A}}{\rho(1-\alpha) \mathcal{A}(1+\mathcal{A})}\right) \frac{\partial \mathcal{A}}{\partial u}+\frac{1}{u-d}\right] \\
& +\frac{\beta^{\frac{\alpha}{\rho(1-\alpha)}} \mathcal{A}^{\frac{\rho-\alpha}{\rho(1-\alpha)}} W}{(1-\alpha)(u-d)(1+\mathcal{A})}\left(\frac{\zeta_{d}^{\frac{1}{\alpha-1}}}{p_{d}}+\frac{\zeta_{u}^{\frac{1}{\alpha-1}}}{p_{u}}\right) \frac{\partial p}{\partial u}
\end{aligned}
$$

If $\rho<0$ (EIS $<1$ ), the sign of $(\rho-\alpha+\alpha(\rho-1) \mathcal{A}) / \rho$ is not determined and thus the sign of total effect is ambiguous. But with large risk aversion, the total effect is positive. If $0<\rho<1$ (EIS $>1$ ), the total effect is positive.

\begin{tabular}{|c|c|c|c|}
\hline Case & $\begin{array}{c}\text { Sub. } \\
\text { effect }\end{array}$ & $\begin{array}{c}\text { Income } \\
\text { effect }\end{array}$ & $\begin{array}{c}\text { Total } \\
\text { effect }\end{array}$ \\
\hline EIS $<1$ & $+/-$ & + & $+/-$ \\
EIS $>1$ & $+/-$ & + & + \\
\hline
\end{tabular}

TABLE 11. The signs of the substitution and income effects for $\partial \theta_{s}^{* m} / \partial u$

Next we derive the Slutsky equation for the effect on the investment in the bond.

$$
\begin{aligned}
\frac{\partial \theta_{b}^{* m}}{\partial u}= & \frac{\partial \theta_{b}^{* h}}{\partial u}-\frac{\partial \theta_{b}^{* m}}{\partial W} \frac{\partial E}{\partial u} \\
= & \frac{\left(u \zeta_{d}^{\frac{1}{\alpha-1}}-d \zeta_{u}^{\frac{1}{\alpha-1}}\right) \beta^{\frac{\alpha}{\rho(1-\alpha)}} \mathcal{A}^{\frac{\rho-\alpha}{\rho(1-\alpha)}} \bar{u}}{R(u-d)(1+\mathcal{A})^{\frac{1}{\rho}}}\left[\left(\frac{\rho-\alpha+(\rho-1) \mathcal{A}}{\rho(1-\alpha) \mathcal{A}(1+\mathcal{A})}\right) \frac{\partial \mathcal{A}}{\partial u}+\frac{1}{u-d}\right] \\
& -\frac{\beta^{\frac{\alpha}{\rho(1-\alpha)}} \mathcal{A}^{\frac{\rho-\alpha}{\rho(1-\alpha)}} \bar{u}}{(1-\alpha) R(u-d)(1+\mathcal{A})^{\frac{1}{\rho}}}\left(\frac{d \zeta_{u}^{\frac{1}{\alpha-1}}}{p_{u}}+\frac{u \zeta_{d}^{\frac{1}{\alpha-1}}}{p_{d}}\right) \frac{\partial p}{\partial u}+\frac{\beta^{\frac{\alpha}{\rho(1-\alpha)}} \mathcal{A}^{\frac{\rho-\alpha}{\rho(1-\alpha)}} \zeta_{d}^{\frac{1}{\alpha-1}} \bar{u}}{R(u-d)(1+\mathcal{A})^{\frac{1}{\rho}}} \\
& -\frac{\left(\zeta_{d}^{\frac{1}{\alpha-1}}-\zeta_{u}^{\frac{1}{\alpha-1}}\right)\left(u \zeta_{d}^{\frac{1}{\alpha-1}}-d \zeta_{u}^{\frac{1}{\alpha-1}}\right) \beta^{\frac{2 \alpha}{\rho(1-\alpha)}} \mathcal{A}^{\frac{2(\rho-\alpha)}{\rho(1-\alpha)}} W}{R(u-d)(1+\mathcal{A})^{2}} \frac{\partial p}{\partial u} .
\end{aligned}
$$

The first three terms show the substitution effect and the fourth term displays the income effect. The sign of each effect is ambiguous. 
We can derive the total effect on the investment in the stock as follows

$$
\begin{aligned}
\frac{\partial \theta_{b}^{* m}}{\partial u}= & \frac{\left(u \zeta_{d}^{\frac{1}{\alpha-1}}-d \zeta_{u}^{\frac{1}{\alpha-1}}\right) \beta^{\frac{\alpha}{\rho(1-\alpha)}} \mathcal{A}^{\frac{\rho-\alpha}{\rho(1-\alpha)}} W}{R(u-d)(1+\mathcal{A})}\left[\left(\frac{\rho-\alpha+\alpha(\rho-1) \mathcal{A}}{\rho(1-\alpha) \mathcal{A}(1+\mathcal{A})}\right) \frac{\partial \mathcal{A}}{\partial u}+\frac{1}{u-d}\right] \\
& -\frac{\beta^{\frac{\alpha}{\rho(1-\alpha)}} \mathcal{A}^{\frac{\rho-\alpha}{\rho(1-\alpha)}} W}{(1-\alpha) R(u-d)(1+\mathcal{A})}\left(\frac{d \zeta_{u}^{\frac{1}{\alpha-1}}}{p_{u}}+\frac{u \zeta_{d}^{\frac{1}{\alpha-1}}}{p_{d}}\right) \frac{\partial p}{\partial u}+\frac{\beta^{\frac{\alpha}{\rho(1-\alpha)}} \mathcal{A}^{\frac{\rho-\alpha}{\rho(1-\alpha)}} \zeta_{d}^{\frac{1}{\alpha-1}} W}{R(u-d)(1+\mathcal{A})} .
\end{aligned}
$$

The sign of the total effect is again ambiguous.

\begin{tabular}{|c|c|c|c|}
\hline Case & $\begin{array}{c}\text { Sub. } \\
\text { effect }\end{array}$ & $\begin{array}{c}\text { Income } \\
\text { effect }\end{array}$ & $\begin{array}{c}\text { Total } \\
\text { effect }\end{array}$ \\
\hline EIS $<1$ & $+/-$ & $+/-$ & $+/-$ \\
EIS $>1$ & $+/-$ & $+/-$ & $+/-$ \\
\hline
\end{tabular}

TABLE 12. The signs of the substitution and income effects for $\partial \theta_{b}^{* m} / \partial u$

The effect of a change in $d$ on the agent's portfolio choice is similar to that of $u$.

\section{CONCLUSION}

We have investigated the effects of changes in the interest rate and the return on the risky asset on an economic agent's current and future consumptions and portfolio choice when the agent has a utility function of the Epstein and Zin type. We have derived the Slutsky equations and decomposed the total effects of changes into the substitution effects and the income effects. We have identified the role of the elasticity of intertemporal substitution and the coefficient of relative risk aversion.

\section{REFERENCES}

[1] G. Pye, Portfolio Selection and Security Prices, Review of Economics and Statistics, 49 (1967), 111-115.

[2] G. Bierwag and M. Grove, Slutsky Equations for Assets, Journal of Political Economy, 76 (1968), 114-126.

[3] A. Sandmo, Capital Risk, Consumption and Portfolio Choice, Econometrica, 37 (1969), 586-599.

[4] A. Sandmo, Portfolio Theory, Asset Dynamics and Taxation: Comparative Statics with Many Assets, The Review of Economic Studies, 44 (1977), 369-379.

[5] S. Fischer, Assets, Contingent Commodities, and the Slutsky Equations, Econometrica, 40 (1972), 371-385.

[6] A. Dalal, Comparative Statics and Asset Substitutability/Complementarity in a Portfolio Model: A Dual Approach, The Review of Economic Studies, 50 (1983), 355-367.

[7] T. Eichner, Mean Variance Vulnerability, Management Science, 54 (2008), 586-593.

[8] T. Eichner, Portfolio Selection and Duality under Mean Variance Preferences, Insurance: Mathematics and Economics, 48 (2011), 146-152.

[9] L. Epstein and S. Zin, Substitution, Risk Aversion, and the Temporal Behavior of Consumption and Asset Returns: A Theoretical Framework, Econometrica, 57 (1989), 937-969.

[10] P. Cook, A 'One Line’ Proof of the Slutsky Equation, American Economic Review, 62 (1972), 139. 